\title{
Mga wika sa sining ng kulit: Implikasyon sa kultura at pagtuturo
}

Augusto, William Jr., S. $\triangle$

Cebu Normal University, Philippines (augustow@cnu.edu.ph)

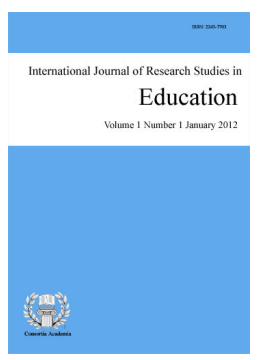

Accepted: 13 January 2021

ISSN: 2243-7703 Online ISSN: 2243-7711

OPEN ACCESS

\section{Abstract}

The study aimed to analyze the language register, syntax structure of the process of sculpture and reasons why the art of sculpture has slowly forgotten in Lapu-Lapu City, Cebu. Moreover, in examining the data, descriptive analysis was used. It includes listing, defining, analyzing and comparing of data. Structural analysis was also used in this study to examine the language structure of sculpture. There were 5 participants who participated in this study who were chosen purposively. From the analysis, the following were discovered: (a) language is formed base on the lifestyle of the people in a community; (b) Cebuano language has different register and syntax structures that were identified in the process of sculpture compared to Tagalog; (c) sculpture like any other art form has also processes to follow; (d) culture is also affected by the changes in the community. This result of the study implied that language serves as life of culture and culture gives life and image to culture. Thus, it is recommended by this study to have integration of local language and culture in the field of teaching to make it contextualize. In addition, giving of training, workshop and exhibit related to sculpture are recommended.

Keywords: kultura; kulit; sining; wika; estruktura 


\section{Mga wika sa sining ng kulit: Implikasyon sa kultura at pagtuturo}

\section{Introduksyon}

Bawat lugar sa Pilipinas ay may kanya-kanyang wika tulad ng Sebwano, Waray, Hiligaynon, Ilokano at iba pa na nagbibigay pagkakilanlan sa kulturang umuusbong sa iba't ibang disiplina sa lipunan tulad ng paniniwala, tradisyon, gawain, sining at iba. Ang wika ay isang masistemang balangkas na sinasalitang tunog na pinili at kabilang o kasapi sa isang kultura o lipunan (Bernales, 2009). Ibig sabihin, ang wika ay nabubuhay sa isang lugar kung saan ito ginagamit at kung saan ang mga tao ay may kulturang sinusunod. Sa kabilang banda naman, ang kultura ay ang pangkabuoang pananaw ng mga tao sa isang lipunan sa mundo at sa kanilang kapaligiran. Ang pananaw na ito ay hango sa paniniwala, tradisyon, sining, uri ng pamumuhay at iba pang mga bagay na nag-uugnay sa kanila at nagpapatibay sa bigkis ng pagkakaisa na siyang nagpapalaganap ng kanilang pangkalahatang diwa, pananaw, kaugalian at adhikain (Mabilin, 2012).

Napalaki ng ginagampanan ng wika at kultura sa pang-araw-araw na buhay ng mga tao. Ito ang nagsisilbing batas ng mga tao na bahagi na sa pamumuhay nila (Almario, 2013). Ito ang nagbibigay sigla sa isang lipunan. Nagkakaroon ng katawagan ang mga bagay-bagay at ang isang kultura dahil sa wika ngunit kung ang mga ito ay hindi na ginagamit, unti-unting nawawala o namamatay ang mga katawagang ito. Ito ang sinapit ng isa sa mga sining na naging pamumuhay ng mga tao sa lungsod ng Lapu-Lapu, ang kulit o paglililok. Ang sining na ito ay unti-unti ng nawawala sa nasabing lugar. Kaya, ito ang nag-udyok sa mananaliksik na gawan ito ng pag-aaral para masuri at maitala ang mga wikang ginagamit nito para ito ay mapreserba at hindi maglaho.

Ang sining ng kulit o paglililok ay hindi lamang hanapbuhay kundi naging bahagi na rin ng kasaysayan at kultura ng Pilipinas. Ang paglililok ay isa sa mga sangay ng Sining Biswal na naisasagawa sa tatlong dimensyon. Ang proseso ng panlililok ay karaniwang ginagamit ang pag-uukit (ang pagtanggal ng materyal) at paghuhulma, bato, metal, seramiko, at kahoy at iba pang materyales upang ito ay maging matibay (Coleo, 2003). Ilan sa mga kilalang manlililok ng bansa ay sina Napoleon Abueva at Eduardo Castillo.

Ang pag-aaral na ito ay nakatuon sa sining ng paglililok gamit ang kahoy sa siyudad ng Lapu-Lapu. Ang siyudad ng Lapu-Lapu ay isang maliit at maunlad na lugar na bahagi ng probinsya ng Cebu. Pangunahing hanapbuhay ng tao rito ay ang industriyal, iilang agrikultural (tulad ng pangingisda), turismo at iba. Isa sa mga umusbong na pagkukuhanan ng hanap buhay sa nasabing lugar ay ang sining ng paglililok. Lumakas ang gawaing ito noong huling bahagi ng dekada 80 hanggang sa unang bahagi ng 2000. Hanggang sa kasalukuyan ay mayroon pa nito ngunit unti-unti ng nawawala dahil sa pag-usbong ng makabagong kagamitan tulad ng fiber cast, turismo at iba pang mas madaling pagkakakitaan.

Ang unti-unting pagkawala ng sining ay isang suliranin sapagkat unti-unti ring namamatay ang wika ng lugar na mahalaga sa pagkakilanlan ng nito (Arrogante, 2002). Wika at kultura ng lugar ang nawawala. Ayon kay Santos (2010), matalik na magkaugnay ang wika at kultura kaya nga naririnig natin na magkabuhol ang wika at kultura. Hindi maaaring paghiwalayin ang wika at kultura. Ang pagkawala o pagkamatay ng isang wika ay nangangahulugan din ng pagkamatay o pagkawala ng isang kultura. Ang wika ang siyang pagkakakilanlan ng isang kultura.

Nilayon ng pag-aaral na ito na masuri ang wika ng paglililok at mga kadahilanan nang paghina ng sining ito. Sinuri rin sap ag-aaral na ito ang sintaktikong kayarian ng proseso ng paglilok at nagkaroon ng paghahambing sa sintaks ng Tagalog. Mahalaga ang pag-aaral na ito sapagkat nagbibigay-diin ito upang maipreserba ang kultura ng bawat lugar. Nagsisilbi rin itong pamukaw sa mga kabataan hinggil sa sining noon. Dagdag pa nito, mahalaga rin itong sanggunian ng mga guro sa kanilang kontekstuwalisadong pagtuturo. 


\subsection{Teoryang sosyo-kultural}

Ang Wika ay isang mahalagang sangkap upang mapanatiling buhay ang iba't ibang kultura ng daigdig. Upang higit na mapagtibay ang pag-aaral na ito, inilalahad sa ibaba ang iba't ibang kaugnay na pag-aaral at literatura. Ang pag-aaral na ito ay nakaangkla sa teoryang Sosyolinggwistiko. Ang wika ay nabubuhay sa iisang kultura at ang kultura ay nabubuhay dahil wika. Ito ay ang paniniwala ng teoryang sosyolinggiwistiko. Pamamalagay ng teoryang ito na ang wika ay isang panlipunang penomenon. Nagiging makabuluhan ang anumang pahayag, aksyon, salita ng isang indibidwal kung ito ay nakakonteksto sa loob ng lipunan at sinasabi sa ibang indibidwal o grupo. At dahil dito, nakabubuo ng iba't ibang konteksto ang paggamit ng wika dahil sa iba't ibang gawain, papel, interes, saloobin, pananaw ang kasangkot sa komunikasyon.

Malaki ang papel ng lipunan sa pagkabuhay ng wika sa kulit o paglililok. Naisasasad din dito ang rehistro ng mga wikang ito at ang paraan ng pagkabuo ng mga salita na makukuha sa proseso ng paglililok. Ayon kay Constantino et al. (1996) sa aklat ni Santos (2010), ang sosyolinggwistikong teorya ay ang ideya ng paggamit ng heterogenous ng wika dahil sa magkakaibang mga indibidwal at grupo na may magkakaibang lugar na tinitirahan, interes, gawain, pinag-aaralan at iba pa. Pinaniniwalaan dito na ang wika ay hindi isang simpleng instrumento ng komunikasyon na ginagamit ng indibidwal ayon sa isang sistemang mga alituntunin kundi isang kolektibong pwersa, isang pagsama-sama ng mga anyo sa isang nagkakaibang cultural at sosyal na mga gawain at grupo. Ayon naman sa pagtatalakay sa dyornal na inilathala ng Shiffield Academy sa United Kingdom, ang sosyolinggwistika ay ang pinagsamang pag-aaral ng sosyolohikal at linggwistika na kung saan pinag-uugnay ang wika at ang lipunan:

\section{"Sociolinguistics is the study of the relationship between language and society. Sociolinguistics can help us understand why we speak differently in various social contexts, and help uncover the social relationships in a community."}

Para naman kay De Saussure (1915), ang wika ay hindi kompleto sa sinumang indibidwal o nagsasalita, nagagawa lamang ito sa loob ng isang kolektibo o pangkat. Gayundin makikita ang paghahalo ng mga varayti ng mga wika, diyalekto, register sa dalawang paraan: a) code switching o palit koda at b) panghihiram. Ang panghihiram ay isang paraan kung saan nakakahalo ang mga varayti. Sa paraang, ang isang salita o higit pa at hinihiram mula sa isang varayti tungo sa isa pang varayti dahil sa walang katumbas ang mga ito sa varayting ginagamit ng nagsasalita. Tinatawag itong lexical borrowing.

Mahalaga ang wika sa isang bayan, dahil ito ay ang anumang binibigkas o isinulat ng tao upang maipahayag ang kanyang saloobin. Ang wika ang siyang nagbibigay pagkakataon para sa mga tao sa iba't ibang lugar upang makapag-usap, upang magkaintidihan at upang makabuo ng pagkakaisa. Ang kultura naman ay ang mga bagay na tumutukoy sa pangkalahatang gawain o aktibidad ng isang tao sa isang lugar (Smith, 2015).

\subsection{Layunin ng pag-aaral}

Layunin ng pag-aaral na ito na masuri at mailahad ang mga rehistro ng wika, sintaktikong kayarian ng proseso ng paglililok at dahilan sa paghina ng sining na ito. Sinuri rin dito ang bahagi ng pananalitang kinabibilangan ng mga wika sa kulit o paglililok at paraan ng pagkabuo ng mga salita.

\section{Metodolohiya}

\subsection{Disenyo ng pag-aaral}

Ang pag-aaral na ito ay ginamitan ng disenyong kwalitatibo. Sa pagsusuri sa datos ng pag-aaral ginamit ang pamaraan ni Creswell (2009). Ang mga kaganapan sa pag-aaral na ginagamitan na ito ay kinapapalooban ng pagtatala, paglalarawan, pagpapakahulugan, pagsusuri at paghahambing. Layunin ng ganitong disenyo na 
sisitematikong mailarawan ang sitwasyon at kundisyon nang makatotohanan at buong katiyakan. Ito ay binubuo ng obserbasyon, pagsasagawa ng sarbey at panayam (Meriam at Tisdell, 2015).

Sa pagsusuri sa kayarian ng wika, ginamitan ito ng descriptive linguistic kung saan sinuri rito ang estruktural na kayarian ng wika. Sa descriptive linguistic na pagsusuri hinihimay-himay ang estruktura ng wika upang lubusang malaman ang ugat nito ang kahulugan (Augusto, 2018). Ang mga partisipante ng pag-aaral na ito ay pinili gamit ang purposive sampling design. Sa ganitong uri ng sampling, pinipili ng mananaliksik ang mga partisipante ayon sa pangangailangan ng pag-aaral (Black, 2010). Ang napiling partisipante ang magiging presentasyon ng buong populasyon ng pag-aaral. Pinili ang mga partisipante ng pag-aaral na ito ayon sa sumusunod na inklusibong kriterya: (a) nakapagtrabaho sa kulit o paglililok; (b) may karanasan sa trabahong kulit sa loob ng dalawang taon pataas; (c) boluntaryong makikilahok sa pag-aaral ng walang pagdadalawang-isip.

Ang instrumentong ginamit ay binubuo ng dalawang bahagi - ang unang bahagi ay may kaugnayan sa personal na propayl ng mga kalahok at isang pangunahing open-ended question na may dalawang sub-kategoryang tanong: (1) Ano-ano ang mga kagamitang kakailanganin sa kulit o paglililok? (2) Ano-ano ang mga proseso nito? (3) Ano ang dahilan ng pagtigil mo sa trabahong ito? Samantala, ang ikalawang bahagi ay ang panayam. Ang kanilang tugon ay inirekord gamit ang awdyo-teyp. Ginawa ang panayam sa mga partisipante sa libreng oras nila. Ang mga instrumentong ito ay ipanabalida sa isang propesor na nagtuturo ng doktorado at mga instraktor na nagtuturo ng wika, panitikan at pananaliksik.Nabuo ang katanungan base sa nakitang pangangailangan at problemang nakita ng mananaliksik.

\subsection{Pinanggalingan ng datos}

Sa pagkamit ng mga layunin ng pag-aaral, limang tigkulit o manlililok ang kinapanayam ng mananaliksik. Isinaalang-alang ang etikal na konsiderasyon sa pamamagitan ng paghingi ng pahintulot sa mga partisipante. Binigyan sila ng consent form bago naganap ang panayam. Pinalitan ng titik (A, B, C, D, E) ang mga pangalan ng kalahok upang mapanatiling confidential ang kanilang pagkakakilanlan. Bago isinagawa ang panayam ay ipinaliwanag muna sa mga partisipante ang layunin ng pag-aaral. Kasunod nito ang pakikipanayam kung saan inirekord ang kanilang mga sagot gamit ang awdyo-teyp. Matapos ang panayam ay nagkaroon ng transkripsyon sa kanilang mga sagot. Bilang pagsiguro sa data privacy, matapos ang buong transkripsyon ng awdyoteyp ng mga partisipante ay binura ang mga ito. Sinundan ang transkripsyon ng pag-analisa sa mga datos gamit ang deskriptibong pagsusuri at pagsusuring estruktural sa mga wikang ginamit.

Sa pagsusuri sa datos ng pag-aaral na ito, ginamit ang pamamaraan ni Creswell (2009) sa pagsusuri ng datos na kinapapalooban ng tatlong hakbang: (1) Kinabibilangan ito ng paghahanda at pag-oorganisa ng mga datos. Kasali sa prosesong ito ang pagtranskrayb sa ginawang panayam, pag-scan sa mga materyales at field notes; (2) Kinapapalooban ito sa pagbasa sa kabuoang datos naitranskrayb upang makuha ang kahulugan ng mga ito; at (3) Dito, magaganap ang pagko-koda sa mga salita, pahayag, at detalye mula sa mga datos na nakuha sa panayam. Dito pinapangkat-pangkat ang mga salita ayon sa kategoryang kinabibilangan nito. Maliban dito, upang tiyak na masuri ang estruktura ng mga salita, ginamitan ito ng descriptive linguistics. Sa pagsusuring ito, sinunod ang sumusunod na hakbang: (1) pagtatala sa mga salita; (2) paghihimay-himay sa mga salita ayon sa panlapi at salitang ugat nito; (3) pagtukoy sa bahagi ng pananalitang kinabibilangan nito; (3) pagbibigay kahulugan sa mga salitang ito.

Sa pagsiguro sa balidasyon ng datos, binalikan ang mga kinapinayam ng mananaliksik upang maipabasa ang mga salita at kinalabasan ng pananaliksik kung sumasangk-ayon ba ito sa kanilang karanasan at kaalaman hinggil sa paglililok.

\subsection{Limitasyon ng pag-aaral}

Saklaw ng pag-aaral na ito ang pagsusuri rehistro, proseso at dahilan ng paghina ng kulit o paglililok sa

16 Consortia Academia Publishing (A partner of Network of Professional Researchers and Educators) 
siyudad ng Lapu-Lapu ng Cebu. Ang datos ng pag-aaral ay limitado lamang sa mga tugon at karanasan ng mga partisipant. Kaya ang pag-aaral ay umiikot lamang sa resulta ng ginawang in-depth interwiews ng mga tigkulit o manlililok.

\section{Resulta at pagtatalakay}

Sa bahaging ito, inilalahad ang proseso ng kulit o paglililok, pagsusuri sa kayarian sa mga wika nito at ang pagbibigay kahulugan sa mga salitang ito.

\subsection{Mga rehistro at bahagi ng pananalita ng mga wika ng kulit o paglililok}

Makikita sa talahanayan 1 sa ibaba ang iba't ibang wika o rehistro sa proseso ng kulit o paglililok. Tinukoy din ang bahagi ng pananalitag kinabibilanga ng mga salitang ito. Kinuha ang mga salitang ito sa mga hakbang sa pagsagawa ng gawaing ito na mula sa pakikipanayam.

\section{Talahanayan 1}

Mga rehistro at bahagi ng pananalita ng mga wika ng kulit o paglililok

\begin{tabular}{|c|c|c|c|}
\hline & Mga salita & $\begin{array}{l}\text { Bahagi ng } \\
\text { Pananalita }\end{array}$ & Kahulugan \\
\hline 1. & pagkulit (paglililok) & Pangngalan & Ang proseso ng pag-uukit o paglililok gamit ang kahoy at pait. \\
\hline 2. & pagdibuho (pagguhit) & Pangngalan & $\begin{array}{l}\text { Ito ang pagbuo ng patern sa hulmahang-papel na ginamitan ng } \\
\text { lapis. }\end{array}$ \\
\hline 3. & mangulitay (manlililok) & Pangngalan & Ang tawag sa taong umuukit o nagsagwa sa proseso ng paglililok. \\
\hline 4. & tigib (pait) & Pangngalan & $\begin{array}{l}\text { Kasangkapang ginagamit sa paglililok. Ito ay matutulis na bagay na } \\
\text { ginagamit upang maporma ang hinahangad na obra. } \\
\text { Nagkakaiba-iba ito sa laki depende sa desinyo. }\end{array}$ \\
\hline 5. & Sumbanan & Pangngalan & Ito ang disenyong mapagkokopyahan. \\
\hline 6. & hulmahang-papel & Pangngalan & Ito ang papel na ginuguhitan ng disenyo o patern. \\
\hline 7. & Silak & Pangngalan & Ito ang likidong pampakintab na ginagamit na ipinapahid sa kahoy \\
\hline 8. & Liha & Pangngalan & $\begin{array}{l}\text { papel de-liha na ginagamit upang maging makinis ang tekstyur ng } \\
\text { kahoy }\end{array}$ \\
\hline 9. & pintal (Pintura) & Pangngalan & $\begin{array}{l}\text { Isang malapot na likido na may iba't ibang kulay at ipinapahid sa } \\
\text { mga parte ng inukitan para magkaroon ng kulay at maging } \\
\text { maganda. }\end{array}$ \\
\hline 10. & Martilyo & Pangngalan & Ito ay ang bagay na Pampukpok sa pako at pait. \\
\hline 11. & Kahoy & Pangngalan & Ito ay solidong bagay na ginagamit sa pag-uukit \\
\hline 12. & Disenyo & Pangngalan & Ito ay tumutukoy sa istilo o anyo ng isang obra. \\
\hline 13. & Kulitunon & Pangngalan & Tawag ito sa bagay na nilililok o uukitn \\
\hline 14. & Patern & Pangngalan & Ito ang tawag sa bagay na hulwaran o pinagbabatayan ng disenyo. \\
\hline 15. & ipapilit (Ididikit) & Pandiwa & $\begin{array}{l}\text { Ito ay ang pagpapadikit sa isang bagay gamit ang isang pandikit } \\
\text { tulad ng glue. }\end{array}$ \\
\hline 16. & $\begin{array}{l}\text { tigibon (uukitin o } \\
\text { lililukin) }\end{array}$ & Pandiwa & $\begin{array}{l}\text { Ito ang tawag sa paghiwa sa parte ng kahoy upang ito ay maporma } \\
\text { ayon sa disenyong sinusunod. }\end{array}$ \\
\hline 17. & gikulit (nililok o inukit) & Pandiwa & Tawag sa kilos ng pag-uukit o paglililok. \\
\hline 18. & pasinawon (Papakinisin) & Pandiwa & Ito ang pagpapakinis sa isang bagay gamit ang papel de-liha \\
\hline 19. & pahamison(Papakintabin) & Pandiwa & Ito ang pagpapakintab sa isang bagay gamit ang silak. \\
\hline 20. & Lihaan & Pandiwa & Ito ang paggamit ng papel de-liha para pakinisin ang kahoy \\
\hline 21. & dukdukon & Pandiwa & Pagpalo sa ulo ng pait at pako upang bumaon. \\
\hline 22. & sapsapan & Pandiwa & pagpapanipis sa kahoy gamit ang pait \\
\hline 23. & mugnaon (gagawin) & Pandiwa & Paggawa ng isang bagay \\
\hline 24. & hait (matulis) & Pang-uri & $\begin{array}{l}\text { Tumutukoy sa dulo ng kutsilyo, pait at iba pang bagay na panghiwa } \\
\text { na matalim o matulis. }\end{array}$ \\
\hline 25. & mano-mano & Pang-abay & Ito ay paggawa ng isang bagay gamit ang sariling kamay at lakas \\
\hline
\end{tabular}

Sa pagsusuri sa bawat hakbang, labinlimang (25) salita ang nakalap ng mananaliksik na tiyak na makikita o esensyal sa proseso ng paglililok. Labing-apat (14) sa mga ito ay pangnglan, siyam (9) sa mga ito ay pandiwa, isa (1) ang pang-uri at isa (1) rin ang pang-abay. Inilalahad sa ibaba ang pagsusuri sa mga salitang ito ayon sa pagkagamit nito sa pangungusap. Ipapakita sa ibaba ang mga pangungusap sa wikang Sebwano at ibibigay ang salin nito sa Filipino. Uunahing ilalahad ang mga salitang ginamit bilang pangngalan kasunod ng pandiwa, pang-uri at pang-abay. 
Ipinahayag ni Al-Kufaishi (2004) na mahirap unawain ang mga salita hangga't hindi natin nauunawaan ang mga estruktura nito. Pinaniniwalaan dito na ang kasanayan sa gramatika o estruktura ng wika, mga panlapi at mga salitang-ugat na bumubuo sa mga anumang anyong berbal na pahayag ay kasangkot sa anumang gawain sa paglinang ng kasanayan sa wika at abilidad sa paggamit nito. Para kay Hajjai at Jacaban (2002), ang mga mahihirap na aytem leksikal na mabubuo mula sa mga panlapi at salitang-ugat ay ang pangunahing suliraning kinakaharap ng isang mag-aaral sa pagbasa ng anumang teksto. Kaya, nararapat na matutunan nila ang morpolohiya o palabuuan ng mga salita upang maiparating nang maayos ang kanilang mensahe sa tagatanggap (Bendalan, 2006).

Sa mga estrukturalista naman, inilalarawan nila ang wika na hindi lamang hinuhubog ng kamalayang panlipunan kundi humuhubog din sa kalayaang panlipunan. Nagpapaliwanag sa mga kahalagahang palipunan (social values); nagsusuri sa mga salita sa akda batay sa kaangkupan nito sa lipunan. Dahil dito napakahalaga ng diskurso sa paghubog ng kamalayang panlipunan. Hindi pinahahalagahan nito ang may-akda at akda bilang daloy ng katotohanan. Nakaugat sa paniniwalang ang kahulugan ay maaari lamang mapalitaw kapag ito ay tiningnan sa mas malawak na estruktura---ang estruktura ng wika. Ang kahulugan ay nakapaloob sa sistema ng wika (ang tinatawag na langue) na nakadepende naman sa aktwal na sinasabi o binigkas (ang parole).

Bilang pagbubuod sa mga pahayag sa itaas, ipinapakita lamang nito na ang wika may sinusunod na patern sa pagbuo at ito ay nakabatay sa uri ng pamumuhay at gawain ng mga tao sa lipunan. Nagkakaroon lamang ng kahulugan ang mga bawat salita sa lugar kung saan ito nagmula at ginagamit. Ipinapahiwatig din nito na sa pagtuturo, mahalagang mabigyang-tuon ang klasipikasyon ng mga salita nang magamit ito nang maayos ng mga bata sa tamang konteksto ng pahayag. Tulad ng wika, kailangan ding alamin ng guro ang kultura ng mga mag-aaral nang sa gayon ay mabigyan ito nang angkop na tulong o estratehiya sa pagtuturo.

\subsection{Sintaktikong pagsusuri sa mga salita}

Makikita sa bahaging ito ang sintaktikong pagsusuri sa proseso ng kulit o paglililok kung saan makikita ang mga salitang nasa talahanayan 1. Nagkaroon din ng paghahambing sa bahaging ito ang Wikang Sebuwano at Wikang Tagalog.

\section{Mga pangungusap gamit ang pangngalan}

Pangungusap sa Sebuwano: Una, magpili ug angay na kahoy nga gamiton para sa pagkulit ug sundan kini sa pag-andam sa mga gamit sama sa hulmahang-papel, martilyo, tigib, lapis, liha, ug silak o pintal.

Salin sa Filipino: Una, pipili ng tamang kahoy na gagamitin para sa paglililok at susundan ito sa paghahanda ng mga kagamitan tulad ng hulmahang-papel, martilyo, tigib, lapis, liha, ug silak o pintal

Pangungusap sa Sebuwano: Ikaduha, human sa pag-andam sa mga gamitunon, sugdan na ug pagdibuho sa patern gamit ang hulmahang-papel nga maoy magsilbing disenyo sa kulitunon. Ang patern nga buhaton kay mabuhat sad gikan sa sumbanan nga mga disenyo.

Salin sa Filipino: Ikalawa, pagkatapos ang paghahanda ng mga kagamitan, sisimulan na ang pagguhit sa patern gamit ang hulmahang-papel na magsisilbing disenyo sa uukitin o lililukin. Ang patern na gagawin ay kukopyahin mula sa napiling disenyo.

Pangungusap sa Sebuwano: Mano-mano kining ang pagbuhat niini sa mangulitay gamit ang kusog sa kamot, tigib, ug martilyo.

Salin sa Filipino: Mano-mano gagawin ng manlililok gamit ang puwersa ng kamay, paita at martilyo.

\section{$\underline{\text { Mga Pangungusap Gamit ang Pandiwa }}$}

Pangungusap sa Sebuwano: Ang patern mao ang magsilbing agianan sa tigib ug gamiton sad kini para

18 Consortia Academia Publishing (A partner of Network of Professional Researchers and Educators) 
Mga wika sa sining ng kulit: Implikasyon sa kultura at pagtuturo

sapsapan ang tibuok kahoy para maporma ang mga gikulit nga ginama ra sa kahoy. Lahi-lahi ang gidak-on sa tigib depende sa disenyong gipili.

Salin sa Filipino: Ang patern ay ang magsisilbing daanan ng pait at gagamitin din ito upang panipisin o bawasan ang kapal ng kahoy para mabuo ang inukit o nililok na ginawa mula sa kahoy.

Pangungusap sa Sebuwano: Itungod ang hait nga bahin sa tigib sa patern nga gibuhat ug $\underline{\text { dukdukon }}$ ang tumoy sa tigib gamit ang martilyo para mabangag o maporma ang disenyo nga gustong makita o mabuhat sa kahoy.

Salin sa Filipino: Itutuon ang matulis na bahagi ng pait sa patern na ginawa at pupukpukin ang itaas na bahagi ng pait gamit ang martilyo para maporma ang dosenyong napili.

Pangungusap sa Sebuwano: Kung maporma na ang disenyong gibuhat, pasinawon na kini gamit ang liha. Lihaan ang tibuok kahoy nga nakulit na para musinaw.

Salin sa Filipino: Kung mabubuo na ang disenyog ginawa, pakikinisin na ito gamit ang liha. Lilihaan ito upang maging makinis ang kahoy.

Pangungusap sa Sebuwano: Human sa pagliha, pahamison na ang gikulit gamit ang silak o varnish.

Salin sa Filipino: Pagkatapos sa pagliliha, pakikintabin na ang inukit gamit ang varnish.

\section{Pangungusap gamit ang pang-uri}

Pangungusap sa Sebuwano: Itungod ang hait nga bahin sa tigib sa patern nga gibuhat ug dukdukon ang tumoy sa tigib gamit ang martilyo para mabangag o maporma ang disenyo nga gustong makita o mabuhat sa kahoy.

Salin sa Filipino: Itutuon ang matulis na bahagi ng pait sa patern na ginawa at pupukpukin ang itaas na bahagi ng pait gamit ang martilyo para maporma ang dosenyong napili.

\section{Pangungusap gamit ang pang-abay}

Pangungusap sa Sebuwano: Mano-mano kining ang pagbuhat niini sa mangulitay gamit ang kusog sa kamot, tigib, ug martilyo.

Salin sa Filipino: Mano-mano gagawin ng manlililok paglililok gamit ang puwersa ng kamay, paita at martilyo.

Mapapansin sa mga sinalungguhitang salita nang isinalin na ito sa wikang Filipino mula sa wikang Sebuwano ay nagbago ang katumbas ng karamihan sa mga salita tulad ng tigib, pintal, silak, mugnaon,ipapiliyt, pagdibuho, kulitunon, sumbanan, sapsapan, pahamison, pasinawon at mangulitay. May iilan ding nananatili tulad ng kahoy, hulmahang-papel, disenyo at martilyo na mula sa wikang Tagalog. Nanatili rin ang salitang patern na hiram na di-ganap mula sa Ingles at ang mano-mano at liha na mula sa wikang Kastila. Ipinakita lamang na katangian ng Wikang Sebwano ang panghihiram din sa ibang wika tulad ng Tagalog at Kastila. Maisasaad din rito ang baryasyon ng wika na nabubuhay sa bawat lipunan kung saan ito ginagamit. Nagkakaroon talaga ng iba't ibang katawagan ng mga bagay-bagay dahil pagkakaiba ng kultura ng bawat isla na may iba't ibang rehiyunal na wika rin.

Isa sa mga nabanggit na katangian sa pagpapakahulugan ni Gleason ay ang pagiging arbitraryo nito na ibig sabihin na bawat lugar o rehiyon sa Pilipinas o iba pang karatig na bansa sa buong mundo ay may kanya-kanyang wika na napagkasunduan ng mga mamamayan na gumagamit nito. Ang wikang arbitraryo ay napagkakasunduan sa paraan ngpagbigkas, pagbabayba, pagpapakahulugan at paggamit sa pagpapahayag. Kaugnay sa naunang pahayag, sinabi ni Constantino et al. (1996), malaking kinalaman o ugnayan ng wika sa 
lipunan at ang lipunang ito ay kinasasangkutan ng mga tao at ang wika ay nagpapahayag ng espiritu/kaluluwa ng mga tao na bumubuo sa lipunan. Ibig sabihin nito na bawat lipunan ay may kanya-kanyang mga katawagan o wika na nabubuhay dahil sa paraan ng pamumuhay at kultural na aspeto nito.

Ayon kay Eastman (1977) isa sa mga salik na nakaiimpluwensya sa pagkakaroon ng varayti ng wika ay ang heograpikal na aspeto nito. Ito ay naglalarawan o tumutukoy sa pagkakahiwa-hiwalay ng lugar at pagkakaiba-iba ng wika dahil sa kalat-kalat na lokasyon ng mga tagapagsalita. Sa kabilang banda naman, ang mga salitang nakasalungguhit ay ginamit bilang pangngalan, pandiwa, pang-uri at pang-abay sa pangungusap sapagkat tumutukoy ito sa ngalan ng bagay. Lahat ng mga salitang ito ay ang mga bagay na ginagamit sa proseso ng paglililok. Ayon kay Santiago (2003), ang bahagi ng pananalita nahahati sa dalawa, ang pangnilalaman at pangkayarian. Nabibilang sa pangnilalaman ang pangngalan, pang-uri, pandiwa at pang-abay. Dagdag pa nito, sa pansemantikang kahulugan, ang pangngalan ay tumutukoy sa pasalitang simbolong ang tinutukoy ay tao, hayop, bagay, pook, pangyayari, atb. Sa pananaw estruktural, ang pandiwa ay nakilala sa pamamagitan ng mga impleksyon nito sa iba't ibang aspekto ayon sa uri ng kilos na isinasaad nito. sa pamamaraang estruktural, ang pang-uri ay nakikilala dahil sa impleksyong nagaganap dito ayon sa kasidhian at hambingan at sa estruktural na pagpapakahulugan, ang pang-abay ay nakikilala dahil sa kasama nito ng isang pandiwa, pang-uri o isa pang pang-abay na bumubuo ng pararila (Pesirla, 2012).

Ayon kay Brown (2017), ang wika ay binubuo ng mga tunog na wasto at malinaw na binibigkas at sinsatitik sa bawat salita o pahayag upang magkaroon ng epektibong paggamit sa layunin na magkaunawaan at makamit ang tagumpay sa pakikipagtalastasan. Bawat diyalekto ng bansa ay may kanya-kanyang set ng bokabularyo na napapabilang sa bawat bahagi ng pananalita. Ang mga ito ay nabubuhay dahil patuloy itong ginagamit ng tao. Mahalagang mapanatili itong buhay sapagkat kayamanan ito ng isang lugar. Ang pagkamatay ng wika ay ang pagkamatay rin ng kultura (Secretariat, 2013).

Bilang paglalahat, mahininuha sa pagsusuring naganap na ang bawat lugar ay may kanya-kanyang set ng bokabularyo na nabubuo at nagkakaroon ng pagpapakahulugan ayon sa pagkagamit ito ng mga taong nasa loob ng komunidad. Ang mga salitang ito ay pwedeng magbago ang klasipikasyon kung ito ay isasalin. Malaki ang impluwensya ng lugar sa pagkakaroon ng baryasyon ng wika. Ipinapahiwatig nito sa pagtuturo na kailangang maging kontekstuwalisado ang gagawing pagtuturo ng guro. Kailangang ipaliwanag ng guro ang paksang itinuturo sa tulong ng mga wika at kulturang lokal nang sa gayon ay madali itong maunawaan ng mga bata.

\subsection{Pagsusuri sa paraan ng pagkabuo ng mga salita}

Makikita sa bahagi ng Talahanayan 2 ang pagsusuri sa paraan ng pagbuo sa mga rehistro ng wika sa proseso ng paglililok. Inilahad ang mga salitang ito sa loob ng talahanayan. Sa pagsusuring estruktural sa paraan ng pagbuo ng salita, nakita na siyam (9) sa mga salita ay may kayariang payak, tatlo (3) ang hiram, labing-apat (14) ang maylapi at isa (1) naman ang tambalang kayarian. Sa lahat ng mga salita, maylapi ang may pinakamaraming bilang. Sa mga maylaping salita, lima (5) sa mga ito ang may unlapi, pito (7) ang may hulapi at dalawa (2) naman ang kabilaan. Ang sumusunod naman ay mga panlaping ginamit: unalpi: pag-, mang-, ipa-, gi-; hulapi: -an, -on; kabilaan: pa-, -an.

Ang kayarian ng wika ay tumutukoy sa estruktura ng morpema (Bulala, 2006). Ang morpema ay tumutukoy sa pinakamaliit nay unit ng salita. Nagiging payak ang kayarian kung ito ay salitang-ugat lamang at walang nagaganap na pagdaragdag, pag-uulit at paglalapi.Inuulit naman ang kayarian kung may nagaganap na pag-uulit sa pantig, bahagi o sa buong salita. Maylapi ito kapag may mga ikinakabit na panlapi sa bahagi ng salitang ugat na maaaring idugtong sa unahan, gitna, hulihan, kabilaan at laguhan. Panghuli, ang tambalan kung dalawang salita ang pinagsama na nakabubuo ng isa pang salita o nanatili lamang ang kahulugan ng mga salitang pinagsama. 
Mga wika sa sining ng kulit: Implikasyon sa kultura at pagtuturo

Talahanayan 2

Paraan ng pagkabuo ng salita

\begin{tabular}{ll}
\hline Paraan ng pagbuo ng salita & \multicolumn{1}{c}{ Mga salita } \\
\hline Payak & hait, tigib, silak, liha, pintal, martilyo, kahoy \\
Maylapi & pagkulit, pagdibuho, mangulitay, sumbanan, kulitunon, ipapilit, tigibon, gikulit, pasinawon, \\
& pahamison, lihaan, dukdukon, sapsapan, mugnaon \\
Tambalan & hulmahang-papel \\
Hiram & disenyo, patern, mano-mano \\
\hline
\end{tabular}

Ang mga kayarian ng salita ay nabubuo sa pagsasasama sa sangkap nito. Isa itong sistematikong pagsasaayos ng mga tunog o ponema na nagbibigay ng isang makabuluhang kahulugan. Ayon kay Santiago (2003) sipi sa pag-aaral ni Concui (2007), ang linggwistika ay makaagham na pag-aaral ng wika. Ang mga ito ay ang ponolohiya na tumutukoy sa pag-aaral ng tunog, morpolohiya na tumutukoy sa pagbuo ng mga salita, sintaksis na tumutukoy sa pag-uugnay-ugnay ng mga salita para makabuo ng pangungusap at ang semantika na tumutukoy sa pag-aaral ng kahulugang nabuo sa salita o pangungusap. Napapaloob dito ang pagtatalakay ng estruktura, katangian, pag-unlad, at iba pang bagay na may kinalaman sa isang wika at ang kaugnayan nito sa iba pang wika.

Maliban sa pagkuha sa salitang-ugat, paglalapi, pag-uulit at pagtatambal ng mga salita, nagkakaroon din ng panghihiram ang isang wika. Ayon kina Legaspi, Luces, Tolosa, at Mindo (2014), may mga salitang o kaya ay hinango sa ibang wika na ngayo'y bahagi nan g ating talasalitaan. Hindi maiwasan ang panghihiram. Maaaring binabago ang baybay ng salita at ang iba naman ay hinihiram ng ganap.Ginagawa ang panghihiram nang ganap upang hindi mabago ang kahulugan ng mga salita lalo na kung wala itong katumbas sa ibang wika. Ganap na hinihiram ang mga salita upang maiwasan ang pagkalito.

Bilang paglalahat, maipapakita sa mga nakalap na datos na dinamiko ang wika sapagkat patuloy itong nagbabago, nanghihihiram at sumasabay sa panahon. Ipinapahiwatig din nito na ang bawat wika ay may kanya-kanyang estruturang sinusunod. Kailangan ding isipin ng guro sa kanyang pagtuturo na ang mga bata rin ay dinamiko kung saan araw-araw iba-iba ang pangangailangan nito at iba-iba ang lebel ng interes kaya mahalaga magkaroon ang guro ng iba't ibang estratehiya at gawain para sa mga mag-aaral. Kailangan din ang guro ay hindi nagpapaiwan sa panahon. Mahalagang sumabay ang guro sa bawat henerasyon ng mga batang makakasalamuha.

\subsection{Proseso sa tradisyunal na paglililok at aspetong pangkultural}

Sa Talahanayan 3 ay makikita ang proseso ng paglililok na isang sining na kailangang paghirapan upang makita ang inaasam na obra. Sa paglikha sa obra, may mga hakbang o proseso itong dinadaanan. Mula sa panayam, nakalap ng mananaliksik ang mga proseso ng paglililok na makikita sa talahanayan 3.

\section{Talahanayan 3}

Proseso sa paglililok ng kahoy

Una, magpili ug angay na kahoy nga gamiton para sa pagkulit ug sundan kini sa pag-andam sa mga gamit sama sa hulmahang-papel, martilyo, tigib, lapis, liha ug silak o pintal.

"Ikaduha, human sa pag-andam sa mga gamitunon, sugdan na ug pagdibuho sa patern gamit ang hulmahang-papel nga maoy magsilbing disenyo sa kulitunon. Ang patern nga buhaton kay mabuhat sad gikan sa sumbanan nga mga disenyo”.

"Ikatulo, ipapilit ang hulmahang-papel nga naay patern sa kahoy nga gamiton sa pagkulit. Ang patern mao ang magsilbing agianan sa tigib ug gamiton sad kini para sapsapan ang tibuok kahoy para maporma ang mga gikulit nga ginama ra sa kahoy. Lahi-lahi ang gidak-on sa tigib depende sa disenyong gipili. "

"Ikaupat, sugdan na ang pagkulit gamit ang tigib. Manomano kining ang pagbuhat niini sa mangulitay gamit ang kusog sa kamot,

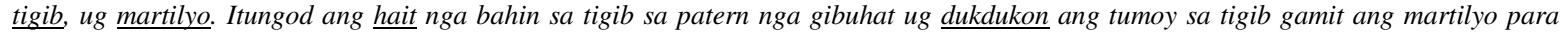
mabangag o maporma ang disenyo nga gustong makita o mabuhat sa kahoy. Tigibon ang tawag sa paggamit sa tigib sa paglugit sa kahoy."

"Ikalima, kung maporma na ang disenyong gibuhat, pasinawon na kini gamit ang liha. Lihaan ang tibuok kahoy nga nakulit na para musinaw.”

"Ikaunom, human sa pagliha, pahamison na ang gikulit gamit ang silak o varnish o kundi puwede sad mugamit ug pintal. Inig human ani paughon para itaud sa lamisa, bangko, divider ug uban pa." 
Sa bawat gawain ay may mga kagamitang gagamitin. Ito ang isinasaad sa unang hakbang kung saan kailangang ihanda tulad ng tracing paper, martilyo, tigib, papel de liha, lagare, varnish at pintura upang maisagawa nang maayos ang gawain ng paglililok. Mahalaga ang mga kagamitang nabanggit upang maging matagumpay at maging maganda ang kalalabasan ng lilok. Matapos maihanda ang mga kagamitang kakailanganin, sisimulan na ang pagguhit sa patern gamit ang lapis at tracing paper. Ang patern ay ang ginuhit mula sa disenyong nagustuhan o napili. Ang kagandahan ng gawang lilok ay nakadepende sa napiling disenyo at pagkagawa ng patern.

Kasunod ng pagguhit ng patern sa tracing paper, ididikit ang tracing paper sa kahoy na uukitin. Ang guhit sa papel na ito ang siyang magsisilbing gabay sa manlililok kung saan padadaanin o itutusok ang kagamitang pang-ukit o tigib upang mabuo ang inaasahang disenyo nito. Iba-iba ang laki ng tigib na gagamitin depende sa disenyong napili. Matapos maidikit ang tracing paper, sisimulan na ang aktwal na paglililok gamit ang puwersa ng kamay ng manlililok, pait, at martilyo. Itutuon ang dulo ng pait sa patern na ginawa at pupukpukin ang ulo o pinakataas na bahagi nito upang dumiin sa kahoy at magkaroon ng kaunting butas. Susundan lamang ang patern hanggang sa mabuo ang disenyong napili sa nililok na kahoy. Ito ang pinakamahalagang bahagi ng paglililok kung saan maaari itong gugulin ng higit sa isang araw depende rin sa laki ng kahoy at porma ng disenyo.

Pagkatapos mabuo ang inaasahang disenyo, pakikinisin ito gamit ang papel de liha. Ang papel de-liha ang isang makalumang anyo sa pagpapakinis ng kahoy. Kasunod ng pagliliha, para mapakintab ito, lalagyan ito ng varnish. Maliban sa varnish, maaari ring lagyan ito ng pintura para mas mapaganda pa ito. Patutuyuin ito at kung natuyo na ay ididikit o ilalagay na ito sa mga upuan, misa, divider at iba pa. Ang papel at varnish tumutulong upang maipreserba ang nililok na kahoy. Ang paglililok ay isang sining at isa ring anyo ng pangkabuhayan. Sa siyudad ng Lapu-Lapu, unti-unti ng nawawala ang tradisyonal na anyo ng paglililok gamit ng kahoy dahil na nga sa pag-unlad ng ibang industriya at may mas madali pang mapagkakakitaan ng pera ang mga tao.

Ang sining na ito ay bahagi na ng kultura sa siyudad ng Lapu-Lapu, maraming mga pordukto rito ang likha mula sa paglililok. Karaniwan sa mga produkto mula sa paglililok ay mga disenyo sa upuan, aparador, mesa, rebulto o estatwa at iba pa. Ayon kay Ortiz, et al. (1992) sipi sa aklat ni Reyes (2012), ang paglililok ay isa sa pinakamatandang anyo ng sining. Ang mga sinaunang guhit ay inukit sa mga bato o kahoy. Dagdag pa nito, ginagamit ng mga tao ang sining na ito sa kani-kanilang ritwal upang humingi ng suportang pang-ispiritwal upang bigyan ng mahabang buhay o mabuhay. Ang paggamit ng kahoy bilang kagamitan sa paglililok ay mas madali sapagkat madali itong mahanap at mas malambot ito kaysa sa bato at iba pa. Sa pandaigdigang kasaysayan, isa sa mga kilalang manlililok na gumamit ng kahoy sa paglililok ay si Donatello, isang italyano sa panahon ng Renasiyemento. Inukit niya ang estatwa ni Saint Mary Magdalene na gawa sa kahoy noong 1455.

Ang ilan sa mga kilalang manlililok na Pilipino ay sina Guillermo Tolentino, Napoleon Abueva, Saprid, Castrillo at iba pa. Karaniwan sa kanilang mga ginawa ay landmarks sa iba't ibang lugar sa Pilipinas. (Estolas et al., 2008). Sa pangkalahatan, mahihinuha na ang paglililok ay isang sining na nangangailangan ng lakas at talino upang malikha ang isang magandang obra. Bilang isang sining, mahalagang matutunan ang bawat hakbang o proseso sa paglikha nito hindi lamang upang para sa obra kundi upang maipasa ito sa iba pang henerasyon at mapapanatiling buhay. Ang mga likha mula sa paglililok ay repleksyon sa kultura tulad ng pamumuhay, gawi, tradisyon at paniniwala ng lugar. Ipinapahiwatig din nito sa pagtuturo na mahalaga bilang guro na may sinusunod na mga hakbang upang maging maayos ang daloy at makamit ng mga mag-aaral ang inaasahang kasanayang kailangang matutunan. Ang pagtuturo at kulit ay parehong bahagi ng isang sining kaya kailangang paghusayan.

\subsection{Industriyalisasyon at modernisasyon}

Sa ginawang panayam ng mananaliksik, natuklasan na isa sa mga dahilan kung bakit sila huminto sa ganitong trabaho ay dahil sa paghina ng demand nito. Dumating din ang mga mga makabagong paraan sa paggawa ng kulit na hindi na mano-mano ang pagsagawa. Maliban dito, nakahanap din ang sila ng mas stable na 
Mga wika sa sining ng kulit: Implikasyon sa kultura at pagtuturo

pagkakakitaan. Narito ang ilang pahayag ng mga partisipante:

"Tumigil kami dahil humina ang demand nito at hindi rin kasi araw-araw may gagawin kami. Depende lamang ito sa dami ng proyekto o o-orderen ng mga customer." - pahayag ni partisipante A.

"May mga dumating na kasing bagong mga paraan sa pagkulit sir na di na mano-mano. Di yun gaanong nakakapagod at mas mabilisan pa ang paglikha.” Pahayag ni partisipante C.

"Nakahanap ako ng stable na pagkakakitaan, nakahanap ako ng trabaho sa MEPZA na arawan ang sweldo." - Pahayag ni partisipante D.

Ang mga tradisyunal na gawaing ito ay unti-unti talagang natatabuban dahil sa industriyalisasyon at modernisasyon. Ito ang sanhi rin sa pagkawala ng ilang mga nakagisnang gawain ng mga tao noon. Ayon nga kay Luiz (2015), ang kultura ay naaapektuhan sa proseso ng economic modernization. Napakahalaga ng gampanin ng bawat mamamayan sa pagpapanatili ng kultura. Kung walang magpi-preserba nito ay unti-unti itong maglalaho.Ipinapakit lamang din nito na ang kultura ay talagang naaapektuhan sa pagbabago ng lipunan. Nawawala ang saysay nito kung patuloy itong tinatalikuran ng mga tao at hindi na isinasagawa. Ganito rin ang pagtuturo, naaapektuhan din ito ng panahon kaya bilang guro, mahalagang maging flexible at magkaroon ng bukas na isipan na tanggapin ang mga pagbabagong nagaganap at magaganap sa pagtuturo.

\section{Kongklusyon at rekomendasyon}

Nilayon ng pag-aaral na ito na masuri ang rehistro, sintatikong kayarian ng proseso ng kulit o paglililok at dahilan ng paghina ng sining na ito. Inilahad din ng pag-aaral na ito ang implikasyon ng mga natuklasan sa pagtuturo hindi lamang sa kultura. Nangunguna sa mga rehistro ng kulit o paglililok ang pangngalan. Sa kayarian naman ay nanguna ang paglalapi. Nakita rin sa pag-aaral ang pagkakaroon ng pagkakaiba sa sintaktikong kayarian ng Tagalog at Sebuwano. Malaki rin ang impluwensya ng industriyalisyon at modernisasyon sa paghina ng sining ng kulit o paglililok.

Ang bawat lipunan ng bansa ay natatangi. Maraming kulturang umiiral at nabubuhay na naiba sa iba pang komunidad. Ang mga kulturang ito ay nanatili mula pa sa mga ninuno o sinaunang panahon dahil na naipreserba ito. Ang midyum na nagpapanatili sa tradisyon, paniniwala, sining, gawain at iba pa ay ang wika. Ang wika at kultura ay hindi dapat magkahiwalay sapagkat binibigyang-buhay ng wika ang kultura kaya mahalagang mapangalagaan ang wika ng bawat lugar nang manatiling buhay ang kinaiingatang yaman ng mga kaninunuan.

Sa kasalukuyan, isinusulong ang pagkakaroon ng kontekstwalisadong pagtuturo, ipinakita ng pag-aaral ang kahalagahan nito sa pagpapanatiling buhay ng kultura at pagkakaroon ng koneksyon ng mga mag-aaral sa wika, mga bagay-bagay at sa mismong lipunan kung saan sila nagmula. Mula sa mga natuklasan, inirerekomenda ng mananaliksik ang sumusunod: (a) magkaroon ng pagpaplanong pampagtuturo na kontekstwalisado na nagbibigay-diin sa iba't ibang sining sa lugar tulad ng kulit o paglililok at iba pa; (b) bigyang-diin ang wikang lokal sa pagtuturo sa iba't ibang paaralan; (c) magkakaroon ng exhibit ng mga produkto mula sa kulit o paglililok; at (d) magkaroon ng worksyap para sa gawain ng paglililok bilang pagbabalik-buhay sa gawaing ito. Ipinanunukala rin ng mananaliksik na magsagawa ng mga pag-aaral na magbibigay-tuon sa mga aspektong hindi sakop ng kasalukuyang pag-aaral tulad ng impak ng modernisasyon sa kultura, Epekto ng kontekstwalisasyong pagtuturo sa kultura at iba pa.

Acknowledgement - Bahagi ng papel na ito ay naipresenta sa 2nd International Conference on Philippine and Asian Studies ng Dalubhasaan ng mga Umuusbong na Mag-aaral ng Araling Filipino (DANUM) sa Visayas State University, Baybay City, Leyte, Philippines noong Ika-3 ng Mayo, 2018. 


\section{Mga sanggunian}

Al-Kufaishi, A. (2004). Translation as a learning and teaching strategy. Babel, 50(1), 45-58. https://doi.org/10.1075/babel.50.1.06alk

Almario, V. (2013). Ortograpiyang pambansa [National orthography]. Commission of the Filipino language.

Arrogante, J. A., provide all authors (2002). Pagpapahalagang sining sa Filipino: Pinta, literature, sayaw at musika [Art appreciation in Filipino: Painting, Literatura, dance and music]. National Bookstore.

Augusto Jr, W. S. (2018). Leksikong kultural ng Tagalog at sinugbuanon: Isang analisis [Cultural Lexicon of Tagalog and Cebuano: An analysis]. Malay, 31(1).

Bendalan, N. (2006). Panlinggwistikang pagsusuri sa mga talaarawan ng mga kadete ng Philippine Military Academy [Linguistic Analysis on the Journal of Philippine Military Academy Cadets] (Thesis). Benguet State University, La Trinidad Benguet.

Bernales, R. A. (2009). Komunikasyon sa makabagong panahon [Communication in the new era]. Mutya Publishing House.

Black, D. M. K. (2010). Business statistics for contemporary. Wiley.

Brown, G. (2017). Listening to spoken English. Routledge. https://doi.org/10.4324/9781315538518

Bulala, I. C. (2006). Meta-analysis ng mga tesis at mga disertasyon tungkol sa pagsusuring panlinggwistika sa Filipino [Meta-analysis of theses and dissertations on linguistic analysis in Filipino]. Cebu Normal University, Cebu City.

Concui, F. N. (2007). Pagsusuring morpo-sintaktikng mga awiting novelty sa Filipino [Morpho-syntactic analysis on Filipino novelty songs]. Holy Name University, Bohol.

Constantino, P., \& Monico A. (1996). Mga patnugot. Mga piling diskurso sa wika at lipunan [Editors. Selected discourses on language and society]. University of the Philippines Press.

Creswell, J. W. (2009). Mapping the field of mixed methods research. Publisher.

De Saussure, F. (1915). Course in general linguistics, 120. Publisher.

Eastman, C. (1977). The emergence of an African regional literature: Swahili. African Studies Review, 20(2), 53-61. https://doi.org/10.2307/523652

Estolas, J. provide all authors (2008). Introduction to humanities. National Book Store.

Jacaban, I. (2002). Pahambing na pag-aaral sa kayariang pangwika ng wikang Filipino at Cebuano ayon sa sintaksis [Comparative study on language structure according to its syntax of Filipino and Cebuano]. Cebu Normal University.

Legaspi, R. L., Luces, C. C., Tolosa, M. L., \& Mindo, D. F. (2014). Bagong Filipino tungo sa globalisasyon [New Filipino toward globalization]. Vibal Group.

Luiz, J. M. (2015). The impact of ethno-linguistic fractionalization on cultural measures: Dynamics, endogeneity and modernization. Journal of International Business Studies, 46(9), 1080-1098. https://doi.org/10.1057/jibs.2015.6

Mabilin, E. (2012). Transpormatibong komunikasyon sa akademikong Filipino [Transformative communication in Filipino academics]. Mutya Publishing House.

Merriam, S. B., \& Tisdell, E. J. (2015). Qualitative research: A guide to design and implementation. John Wiley $\&$ Sons.

Pesirla, A. O. (2012). Ang linggwistika sa sugbu-anung binisaya [Cebuano linguistics]. Anvil Publishing House.

Reyes, A. R. (2016). Komunikasyon at pananaliksik sa wika at kulturang Pilipino [Communication and research in the Filipino language and culture]. Diwa Learning.

Santiago, A. (2003). Makabagong balarilang Filipino [New Grammar in Filipino]. Rex Book Store.

Santos, A. L. (2010). Ang akademikong filipino sa komunikasyon [The academic communication in Filipino]. Mutya Publishing House.

Secretariat, D. o.-I. (2013). Sinugbuanong binisaya: Yunit 3 [Sinugbuanong Binisaya: Unit 3].

Smith, S. (2015). A sense of place: Place, culture and tourism. Tourism Recreation Research, 40(2), 220-233. https://doi.org/10.1080/02508281.2015.1049814 